\title{
Assessment of Telomere Length and Molecular Characterization of TERT Gene Promoter in Periampullary Carcinomas
}

\section{Cleandra Gregório}

Universidade Federal do Rio Grande do Sul

\section{Shefali Thakur}

International Agency for Research on Cancer

Raquel Camara Rivero

Universidade Federal do Rio Grande do Sul

Simone Márcia dos Santos Machado

Hospital de Clinicas de Porto Alegre

Cyrille Cuenin

International Agency for Research on Cancer

Christine Carreira

International Agency for Research on Cancer

Valerie White

International Agency for Research on Cancer

lan A Cree

International Agency for Research on Cancer

Katarina Vukojevic

University of Split Faculty of Medicine: Sveuciliste u Splitu Medicinski fakultet

Merica Glavina Durdov

University of Split Faculty of Medicine: Sveuciliste u Splitu Medicinski fakultet

Alessandro Bersch Osvaldt

Universidade Federal do Rio Grande do Sul

Patricia Ashton-Prolla

Universidade Federal do Rio Grande do Sul

\section{Zdenko Herceg}

International Agency for Research on Cancer

Fazlur Rahman Talukdar ( $\nabla$ fazlur08@gmail.com )

International Agency for Research on Cancer https://orcid.org/0000-0002-6558-5543 
Keywords: Periampullary carcinoma, Telomeres, Telomerase, TERT promoter, Relative Telomere Length, TERT expression

Posted Date: December 8th, 2021

DOI: https://doi.org/10.21203/rs.3.rs-1143437/v1

License: (c) (1) This work is licensed under a Creative Commons Attribution 4.0 International License. Read Full License 


\section{Abstract}

Genetic and epigenetic alterations of the telomere maintenance machinery like telomere length and telomerase reverse transcriptase (encoded by TERT gene) are reported in several human malignancies. However, there is limited knowledge on the status of the telomere machinery in periampullary carcinomas (PAC) that are rare and heterogeneous groups of cancers arising from different anatomic sites around the ampulla of Vater. In the current study, we investigated the relative telomere length (RTL) and the most frequent genetic and epigenetic alterations in the TERT promoter in PAC $(\mathrm{n}=20)$ and compared with tumor-adjacent nonpathological duodenum (NDu; $n=16)$. We found shorter RTLs (1.27 vs $1.33, P=0.01)$ and lower TERT protein expression $(p=0.04)$ in PAC tissues as compared to the NDu. Although we did not find any mutation at two reactivating hotspot mutation sites of the TERT promoter, we detected polymorphism in $55 \%(11 / 20)$ of the cases at rs2853669 $(T>C)$. Also, we found a hypermethylated region in the TERT promoter of PACs consisting of four CpGs (cg10896616 with $\Delta \beta 7 \%$; cg02545192 with $\Delta \beta$ $9 \%$; cg03323598 with $\Delta \beta 19 \%$; and cg07285213 with $\Delta \beta 15 \%)$. In conclusion, we identified shorter telomeres with DNA hypermethylation in the TERT promoter region and lower TERT protein expression in PAC tissues. Further studies with a larger sample size are necessary to substantiate these results.

\section{Introduction:}

Periampullary carcinomas (PACs) encompass a heterogeneous group of neoplasms (L. 2001) arising from four distinct anatomic sites proximal to the major duodenal papilla, namely - ampullary adenocarcinoma (AA), distal cholangiocarcinoma (DCC), duodenal adenocarcinoma (DA), and pancreatic head adenocarcinoma (PDAC) (Sarmiento, Nagomey et al. 2001, Albores-Saavedra, Schwartz et al. 2009, Kamarajah 2018, Hester, Dogeas et al. 2019). PACs are a rare malignancy whose incidence rate is difficult to establish as most reported studies only reflect the prevalence of surgically resected cancers (Yeo, Sohn et al. 1998, Riall, Cameron et al. 2006, He, Ahuja et al. 2014, Chandrasegaram, Chiam et al. 2015). However, on average, taken together, they represent $5 \%$ of all gastrointestinal malignancies (Sarmiento, Nagomey et al. 2001, Berberat, Künzli et al. 2009, Kamarajah 2018, Song, Kim et al. 2020). Due to their anatomical proximity, PACs share clinical characteristics and interventions, the most effective of which, regardless of the site of origin, is surgical resection by 'pancreaticoduodenectomy' (PD) (Ohtsuka and Miyazaki 2018). However, even after surgical resection, the long-term survival ( $\geq 5$ years) rate in patients is unsatisfactory ( 20\%) (El Nakeeb, El Sorogy et al. 2018) and varies greatly among the subtypes of PACs (Bramhall, Allum et al. 1995, Neoptolemos, Russell et al. 1997, Poultsides, Huang et al. 2012).

For better clinical outcomes and therapeutic management, it is important to distinguish the site of origin of cancer and thus the tumor identity before deciding on the required intervention. However, the precise site of origin of PACs is usually challenging to determine before the surgical intervention. Thus, the correct preliminary diagnosis is often difficult, as in the case of PAC patients with locally advanced or metastatic disease that impedes the curative surgical resection. For this reason, during the past years, many studies (Ching and Rhodes 1989, Steinberg 1990, Lundin, Roberts et al. 1994, Ritts, Nagorney et al. 1994, Satake and Takeuchi 1994, Kau, Shyr et al. 1999) have established distinct molecular markers of 
PACs, specifically carcinoembryonic antigen (CEA) and carbohydrate antigen 19-9 (CA 19-9), but also CA 242 (Haglund, Lundin et al. 1994), CA 50, CA 12-5, tissue polypeptide antigen (TPA) (Benini, Cavallini et al. 1988) and various mRNAs and miRNAs (Sandhu, Bowitz Lothe et al. 2015). These markers exhibit some diagnostic and prognostic value and can partially explain differing tumor type behavior and patient survival (Forsmark, Lambiase et al. 1994, Okusaka, Okada et al. 1998, Distler, Pilarsky et al. 2013, Park, Shin et al. 2021). However, this is far from enough to fully understand this malignancy and necessitates a more detailed molecular characterization of the disease.

Eukaryotic organisms exhibit highly specialized DNA-protein structures at the end of linear chromosomes, called telomeres; that help maintains genomic stability (Hernandez-Sanchez, Xu et al. 2016). Owing to the end-replication problem, telomeres are progressively shortened during successive cell divisions that cause cellular senescence, ultimately leading to cell mortality. Since cancer is a disease of cellular immortality, it is not surprising that cancer cells need to overcome this senescence by countering telomere shortening (Shay 1995). Cancer cells achieve this with the help of an enzyme called telomerase, which adds telomere repeat sequence to the $3^{\prime}$ end of the telomere, giving them infinite replicative potential (Feng, Funk et al. 1995). The catalytic subunit of telomerase is telomerase reverse transcriptase (TERT) which has been implicated to have a role in human cancer (Kirkpatrick and Mokbel 2001). Approximately 90\% of all human cancers exhibit transcriptional activation of TERT (Holt, Wright et al. 1997) (Shay and Bacchetti 1997). Hundreds of TERT polymorphisms have been found in human cancers, (Mocellin, Verdi et al. 2012) including hot-spot mutation of the gene promoter causing several sequence variations (Bell, Rube et al. 2016, Dratwa, Wysoczańska et al. 2020), which act as pivotal players in the activation of TERT transcription (Yuan, Larsson et al. 2019). In addition, epigenetic modification of the TERT promoter via methylation has been reported in about 50\% of all human cancers (Lee, Leao et al. 2019, Dratwa, Wysoczańska et al. 2020). Despite the available detailed descriptions of the specific mechanism behind telomere maintenance of many cancers, it is still poorly understood in PAC. Thus, we reasoned that the information on the telomeric status and TERT gene features of PAC will help in a better understanding of its molecular landscapes. Following this rationale, we inspected and report the relative telomere length $(R T L)$, as well as the most frequent genetic and epigenetic alterations in the TERT gene, using Sanger sequencing and 450K methylation array. In addition, we inspected TERT gene expression by immunohistochemistry in AA and DA. Lastly, we also explored whether these parameters were correlated with clinicopathological features and patient prognosis. This study constitutes a pilot study in the field of telomere biology in AA and DA and reveals new information about the telomere maintenance in these cancers.

\section{Methods:}

\section{Study samples}

Patients with PAC and DUOAC were recruited between March 2011 and September 2018 from the Hospital de Clínicas de Porto Alegre (HCPA/UFRGS), Brazil. The inclusion criteria were recent pathologyproven diagnosis and no history of previous or current chemo- or radiotherapy treatment. Samples from 
tumor and nonpathological mucosae of the duodenum (NDu) were obtained during surgical pancreatoduodenectomy with curative intent without known residual disease and stored according to the biobank protocols from the hospital. A portion of each tissue sample was frozen and stored at $-80^{\circ} \mathrm{C}$, whereas the remaining tissue was fixed in $10 \%$ buffered formalin to prepare Formalin-Fixed ParaffinEmbedded (FFPE) blocks. The characteristics of the patients were obtained from medical records and the prognostic factors of these cases was published recently (Vilhordo, Gregorio et al. 2021). The study was approved by the Ethics Committee of Hospital de Clínicas de Porto Alegre (Project number: 2014-0526) and conducted following the Declaration of Helsinki. All subjects gave their informed consent for inclusion before they participated in the study.

\section{Pathology}

Tissue sections derived from FFPE blocks were stained with hematoxylin \& eosin to confirm the diagnosis and assess the sample quality. The tumoral and non-tumoral samples were independently examined by two experienced pathologists (R.C.R. and S.M.S.M.), in a blinded manner. The classification of malignant epithelial tumors of the exocrine pancreas recommended by The World Health Organization (WHO) was applied for the histological type(Nagtegaal, Odze et al. 2020). The histopathological grade was based on the extension of the glandular formation, being considered well-differentiated tumors with more than $95 \%$ of glands, moderately differentiated tumors with $50-95 \%$ of glands and poorly differentiated tumors with $49 \%$ or less of glands (Adsay, Basturk et al. 2005). The location of the neoplasia was defined according to the anatomical subdivisions recommended by the AJCC, as follows (Amin, Greene et al. 2017): Tumors of the head of the pancreas are those arising to the right of the left border of the superior mesenteric vein (the uncinate process is part of the head). Tumors of the body of the pancreas are those arising between the left border of the superior mesenteric vein and the left border of the aorta. Tumors of the tail of the pancreas are those arising between the left border of the aorta and the hilum of the spleen.

\section{Sample preparation}

DNA isolation was done from fresh frozen tumor tissues $(n=20)$ and NDu $(n=16)$ using the PureLink Genomic DNA Kit (Thermo Fisher Scientific) according to the manufacturer's protocol. The samples with pathologically $>60 \%$ neoplastic cells were considered as tumor and $100 \%$ nonpathological mucosae of the duodenum was considered normal. The eluted DNA was quantified using Qubit V2.0 (Invitrogen, Carlsbad, USA).

\section{Relative telomere length measurement}

We performed a qRT-PCR based estimation on relative telomere length (RTL) assessment by comparing the abundance of the telomeric template $(T)$ relative to a single-copy gene ( $\mathrm{S}$, here albumin) and expressed as the ratio between T/S (Hosen, Rachakonda et al. 2015). Each reaction was performed in triplicate, in an optical 96-well reaction plate compatible with the Bio-Rad Real-Time PCR Detection System using the SYBR Green Supermix (Bio-Rad, Hercules, CA). The thermal cycling was performed as per the modified Cawthon method (Cawthon 2009). 
The signal acquisition at $74^{\circ} \mathrm{C}$ provided the $\mathrm{Ct}$ values for the amplification of the telomere template and signal acquisition at $88^{\circ} \mathrm{C}$ gave the $\mathrm{Ct}$ values for the albumin template. After the final incubation at $88^{\circ} \mathrm{C}$, the specificity of all amplifications was determined by melting curve analysis. Reactions were then cooled to $55^{\circ} \mathrm{C}$, and the signal was acquired from $55^{\circ} \mathrm{C}$ to $95^{\circ} \mathrm{C}$, in increment $0.5^{\circ} \mathrm{C}$ steps, with a 30 s period per step. PCR conditions and the primers used for the telomere and the albumin are listed in Supplementary Table T1. The quality of PCR amplification was determined by standard curves using reference DNA in triplicates (serial dilutions in the range of $150-5.55 \mathrm{ng}$ ). Telomere and albumin reactions were added in different wells and $\mathrm{Ct}$ values were obtained based on the respective averages.

\section{TERT promoter mutational detection}

The tumor mutational status of the TERT core promoter and the surrounding region was determined by Sanger sequencing (Rachakonda, Hosen et al. 2013), using the Applied Biosystems 3730xl genetic analyzer. Sequencing data was analyzed using the CodonCode Aligner version 7.1 software referring to the sequences from the NCBI gene database, TERT (chr5: 1,295,071-1,295,521, hg19 GRCH37). The primer sequences and PCR conditions for Sanger sequencing are shown in Supplementary Table T2.

\section{TERT promoter DNA methylation estimation}

We retrieved beta $\beta$ values from Illumina Infinium Human Methylation450K Bead-Chips HM450K data (Illumina, San Diego, CA, USA). To investigate the DNA methylation status TERT promoter, we retrieved the normalized $\beta$ values of the probes from the genomic regions (chr5:1,291,235-1,295,737), which included probes in its promoter, gene body, and introns. The $\beta$ values of four $\mathrm{CpG}$ s from this region are shown in Figure 4 with their genomic coordinates.

\section{TERT protein expression}

Tumor samples and their respective surrounding duodenal non-tumoral FFPE tissue were retrieved and included in tissue microarrays (TMAs). TMAs were prepared from formalin-fixed paraffin-embedded tissue sections using a 1-mm punch in triplicate. Immunohistochemistry (IHC) was carried out on 4- $\mu \mathrm{m}$ sections. Briefly, slides were deparaffinized and then rehydrated before the staining procedure can be performed. For the antigen retrieval process, slides were placed in a preheated Epitope Retrieval Solution $\mathrm{pH} 6\left(95^{\circ} \mathrm{C}\right.$ to $\left.99^{\circ} \mathrm{C}\right)$ and maintained at this temperature for 10 minutes followed by a 20-minute cooldown period at room temperature. After a brief rinse in distilled water, slides were immersed in Wash Buffer in preparation for immunostaining.

Specimens were blocked with a peroxidase-blocking reagent for 5 minutes followed by $1 \mathrm{~h}$ incubation with the polyclonal primary antibody anti-telomerase reverse transcriptase (600-401-252S, Rockland Inc., Limerick, PA, USA) at a dilution of 1:1000. Slides were then incubated for 30 minutes with the visualization reagent followed by five minutes incubation with DAB. Slides were subsequently counterstained with hematoxylin, dehydrated, mounted with permanent mounting medium, and coverslipped. All steps, except for the epitope retrieval, were performed at room temperature. Immunoreactivity was visualized by light microscopy and the gradation was done based on slides 
displaying intensities "negative/no staining as 0 ," "low/weak staining as 1," "medium staining 2," or "high staining as 3." Also, the percentage of stained cells was noted for the final score determination. A final score was calculated by multiplying the staining intensity and percentage of positively stained cells, which is described in Supplementary Table T3.

\section{Statistical Analysis:}

The comparison between groups (PAC vs NDu) of the RTL and methylation profile was performed using the Wilcoxon test. Association between clinicopathological factors and RTL and rs2853669 was performed by Mann-Whitney and Fisher's Exact test, respectively. Correlation between RTL and methylation profile was performed by Spearman correlation. Five-year survival rates were calculated by the Kaplan-Meier method, and univariate survival analysis was performed by the Log-rank, Breslow, and Tarone-Ware tests. All statistical analysis was performed with SPSS version 24 (IBM). $p<0.05$ was considered statistically significant.

\section{Results:}

\section{Sample characteristics}

Our study included 20 PAC cases among which 12 were males and 8 were females. Among these, half of our PAC cases (50\%) were from intestinal-type adenocarcinoma. Details of all pathological sub-types are shown in Table $1.30 \%(n=6)$ of the cases were $\geq 65$ years of age and all the cases were from stage II to IV. The tumor grade was moderate or poor differentiation for $95 \%(19 / 20)$ of the cases (Table 1$)$. We also included macroscopically examined tumor-adjacent nonpathological duodenum tissues $(N D u, n=16)$ for comparison.

\section{Relative Telomere Length (RTL)}

We analyzed the RTL of PACs and NDu by comparing the abundance of the telomeric template $(T)$ relative to a single-copy gene (S, here albumin) using qRT-PCR. The RTL is expressed as the ratio between T/S and the higher value will signify longer RTL. The average RTL for PAC tissue was 1.27 (range 1.101.42) and NDu tissue was 1.33 (range 1.2-1.38); $P=0.01$ which signifies shorter telomere length in tumors than the adjacent nonpathological duodenum (Figure 1).

\section{TERT protein expression}

To investigate the TERT protein expression, we compared the immunohistochemistry (IHC) score between PAC and NDu tissues. Negative staining was considered as 0 and three categories of positive staining (C1: 1-3; C2: 4-6 and C3: 7-9) was generated by scoring the intensity and percentage of positively stained cells. The details of the score calculation are specified in Supplementary Table T1. PAC tissues exhibited a greater number of category $C 1$ (55\% vs $20 \%$ ) than NDu tissues, which is associated with weak/poor staining. However, TERT positive expression rate was observed higher in NDu than the PAC for category 
C2 (45\% vs 30\%) and C3 (15\% vs 10\%). Since scores for category C2 and C3 were derived from medium/high staining, it is apparent that TERT protein expression is more prominent in NDu (Table 2).

\section{TERT promoter mutation}

TERT promoter sequencing results revealed no mutation at the two reactivating hotspot mutation sites C228T (NG_009265.1: g.4935C>T) and C250T (NG_009265.1: g.4913C>T) in the PAC tumors (Figure 3 and Supplementary Figure S1). However, within the sequenced region, we observed 11/20 (55\%) cases with polymorphism at rs2853669; T>C (NG_009265.1: g.4814T>C). The polymorphic site is present in the upstream region of the two hotspot mutation sites shown in Figure 3. This polymorphism at rs 2853669 with the two hotspot mutations C228T and C250T is usually associated with an increased risk of developing cancer (Vinothkumar, Arun et al. 2020). Although in our PAC samples we could observe this polymorphism, we did not detect any sequence variation in the two-hotspot region. To investigate further if there is any clinical significance of the identified polymorphic site rs2853669, we performed survival analysis by stratifying cases based on the status of polymorphism (Supplementary Figure S2). Nevertheless, the differences in survival in all patients with and without the rs2853669 polymorphism were not significant $(P=0.80)$.

\section{TERT promoter methylation}

We identified a differentially methylated region in the TERT promoter that was hypermethylated in PAC tissues in comparison with NDu tissues. Seven CpG sites showed this differential methylated trend with a $\triangle \beta$ (DNA methylation difference) of $9 \%$ among which, four $\mathrm{CpGs}$ were significantly hypermethylated in PAC samples. The identified differentially methylated region in the TERT promoter region constitutes multiple transcription factors and related protein binding sites that overlap with the four significantly hypermethylated CpG sites. In these CpGs $\Delta \beta$ at $\operatorname{cg} 10896616$ was $7 \%, P=0.006$; at cg02545192 $\Delta \beta$ was $9 \%, \mathrm{P}=0.005$; at $\operatorname{cg} 03323598 \Delta \beta$ was $19 \%, \mathrm{P}=0.002$, and at $\operatorname{cg} 07285213 \Delta \beta$ was $15 \%, \mathrm{P}=0.04$ (Figure 4).

\section{Discussion:}

In this study, we report shorter RTL in PAC and aimed to investigate molecular features of the TERT gene and its protein expression to understand the possible link with alteration of telomere length (Figure 5). However, it should be noted that telomere lengthening can be independent of TERT gene alterations and alternative mechanisms can lead to variations in RTL (Yuan, Larsson et al. 2019). Here we observed lower TERT protein expression in tumors relative to NDu based on IHC analysis. Previous studies have shown that shorter telomeres are associated with a higher risk for several cancers (Broberg, Björk et al. 2005, Risques, Vaughan et al. 2007, Jang, Choi et al. 2008, Hou, Savage et al. 2009, Xing, Ajani et al. 2009, Mirabello, Garcia-Closas et al. 2010, Willeit, Willeit et al. 2010), including pancreatic cancers (Kobitsu, Tsutsumi et al. 1997, Skinner, Gangnon et al. 2012) (Hong, Heaphy et al. 2011) and hepatocellular carcinomas (Oh, Jo Chae et al. 2003). Truncated telomeres are considered a defining characteristic of most carcinomas (McGrath, Wong et al. 2007). Genetic instability associated with short telomeres is 
described as an early event in tumorigenesis that acts as a predisposition factor for many cancers $\mathrm{Wu}$, Amos et al. 2003). Since telomere shortening is very unlikely to provide any direct growth advantage to the cells, it is presumed to promote cancer progression by causing telomere crisis (van Heek, Meeker et al. 2002), which can induce numerous changes relevant to cancer including chromothripsis, kataegis, and tetraploidization (Maciejowski and de Lange 2017). This is in line with our observation, and it would be interesting to inspect these tumors for chromosomal abnormalities.

While telomere dysfunction provokes chromosomal aberrations and aids initiation of carcinogenesis, telomerase-mediated telomere maintenance enables such predisposed cells to efficiently achieve a fully malignant endpoint, including metastasis (Chang, Khoo et al. 2003). Therefore, telomerase activation by various mechanisms including TERT promoter mutation is common in many cancers, including hepatocellular carcinomas (Huang, Wang et al. 2015). However, these are extremely rare in pancreatic cancers (Vinagre, Nabais et al. 2016, Posch, Hofer-Zeni et al. 2020), but no hotspot mutations were found in our samples. We also observed a polymorphism at rs 2853669 in $55 \%$ of the cases but we could not find any significant correlation with prognosis. A recent study proposed the association of this polymorphism with the two hotspot mutations in increasing the risk of cervical cancer (Vinothkumar, Arun et al. 2020). Nevertheless, there are limited functional studies to establish the role of rs2853669 polymorphism in carcinogenesis.

Then, we aimed to examine if the TERT expression was altered by epigenetic mechanisms in these cancers instead. In previous studies, TERT Hypermethylated Oncological Region (THOR) located in promoter was found to be upregulating TERT expression and associated with poorer clinical outcomes (Castelo-Branco, Leao et al. 2016, Lee, Leao et al. 2019). In line with this, we observed hypermethylation of tumor tissues in a region downstream of THOR and near the transcription start site (TSS). The detected lower TERT protein expression in tumors might be due to the hypermethylated region near the TSS of the TERT promoter. Also, the presence of some transcription factor binding site at the hypermethylated region further substantiate that this could be the possible reason for the downregulation of TERT expression. One of the constraints of this study is that we did not have RNA samples to validate the association of TERT promoter hypermethylation with reduced TERT protein expression in PAC tissues.

In conclusion, we observed shorter RTL and lower TERT protein expression in PAC. We further report a hypermethylated region in TERT promoter and polymorphism at rs 2853669 in $55 \%$ of the cases. The TERT promoter hypermethylation might be linked with shorter RTL and lower TERT protein expression in PAC. Additional studies with a larger sample size could validate these findings in PAC and associate with clinical parameters to improve patient outcomes.

\section{Declarations:}

\section{FUNDING}

The work reported in this article was undertaken by F.R. Talukdar partly during the tenure of a Postdoctoral Fellowship from the International Agency for Research on Cancer (IARC), partially supported 
by the EC FP7 Marie Curie Actions-People- Co-funding of regional, national, and international programs (COFUND). C.Gregório partly during the tenure of a Doctoral studies from the Universidade Federal do Rio Grande do Sul (UFRGS), supported by the Coordenação de Aperfeiçoamento de Pessoal de Nível Superior (CAPES, Brazil- Programa de Doutorado Sanduíche no Exterior- PDSE-, Grant\# 47/2017, process no 88881.198102/2018-01). The work in the Epigenetics Group at IARC is supported by grants from the Institut National du Cancer (INCa, France), the European Commission (EC) Seventh Framework Program (FP7) Translational Cancer Research (TRANSCAN) Framework, the Foundation ARC pour la Recherche sur le Cancer (France), Plan Cancer-Eva-Inserm research grant, La direction 1enerale de l'offre de soins (DGOS), and INSERM (SIRIC LYriCAN, INCa-DGOS-Inserm_12563) to Z. Herceg. Fundo de Incentivo à Pesquisa (FIPE) from Hospital de Clínicas de Porto Alegre (HCPA, Brazil- GPPG Grant \#14-0526), Conselho Nacional de Pesquisa (CNPq, Brazil- Universal Grant \#424646/2016-1).

\section{ACKNOWLEDGEMENTS}

We would like to thank Professor Raymond Dalgleish for helping us with the correct nomenclature for the two hot-spot mutations and polymorphism in the TERT promoter.

\section{COMPETING INTERESTS}

The authors declare no conflict of interest.

\section{IARC DISCLAIMER}

Where authors are identified as personnel of the International Agency for Research on Cancer / World Health Organization, the authors alone are responsible for the views expressed in this article and they do not necessarily represent the decisions, policy, or views of the International Agency for Research on Cancer / World Health Organization.

\section{CONTRIBUTION}

CG: conceptualization, data curation, methodology, formal analysis, validation, writing-original draft preparation and editing; ST: data interpretation, validation, writing-original draft preparation and editing; RCR: methodology, investigation and formal analysis; SMSM: methodology, investigation and formal analysis; CC: methodology; validation; ChC: methodology; validation; VW: methodology, investigation and formal analysis; IC: methodology and investigation; KV: methodology, investigation and formal analysis; MGD: methodology, investigation and formal analysis; ABO: methodology, investigation and formal analysis; PAP: conceptualization, project administration, supervision, investigation, resources, fund acquisition; ZH: conceptualization, project administration, supervision, investigation, resources, fund acquisition; FT: conceptualization, project administration, supervision, investigation, data curation, methodology, formal analysis, validation, writing-original draft preparation and editing. All authors reviewed and edited the manuscript. 
All patients provided informed consent to participate in this study. The study was approved by the Ethics Committee of Hospital de Clínicas de Porto Alegre (Project number: 2014-0526) and conducted following the Declaration of Helsinki.

\section{References:}

1. Adsay, N. V., O. Basturk, M. Bonnett, N. Kilinc, A. A. Andea, J. Feng, M. Che, M. R. Aulicino, E. Levi and J. D. Cheng (2005). "A proposal for a new and more practical grading scheme for pancreatic ductal adenocarcinoma." Am J Surg Pathol 29(6): 724-733.

2. Albores-Saavedra, J., A. M. Schwartz, K. Batich and D. E. Henson (2009). "Cancers of the ampulla of vater: demographics, morphology, and survival based on 5,625 cases from the SEER program." J Surg Oncol 100(7): 598-605.

3. Amin, M. B., F. L. Greene, S. B. Edge, C. C. Compton, J. E. Gershenwald, R. K. Brookland, L. Meyer, D. M. Gress, D. R. Byrd and D. P. Winchester (2017). "The Eighth Edition AJCC Cancer Staging Manual: Continuing to build a bridge from a population-based to a more "personalized" approach to cancer staging." CA Cancer J Clin 67(2): 93-99.

4. Bell, R. J., H. T. Rube, A. Xavier-Magalhães, B. M. Costa, A. Mancini, J. S. Song and J. F. Costello (2016). "Understanding TERT Promoter Mutations: A Common Path to Immortality." Mol Cancer Res 14(4): 315-323.

5. Benini, L., G. Cavallini, D. Zordan, P. Rizzotti, L. Rigo, G. Brocco, L. Perobelli, M. Zanchetta, P. Pederzoli and L. A. Scuro (1988). "A clinical evaluation of monoclonal (CA19-9, CA50, CA12-5) and polyclonal (CEA, TPA) antibody-defined antigens for the diagnosis of pancreatic cancer." Pancreas 3(1): 61-66.

6. Berberat, P. O., B. M. Künzli, A. Gulbinas, T. Ramanauskas, J. Kleeff, M. W. Müller, M. Wagner, H. Friess and M. W. Büchler (2009). "An audit of outcomes of a series of periampullary carcinomas." Eur J Surg Oncol 35(2): 187-191.

7. Bramhall, S. R., W. H. Allum, A. G. Jones, A. Allwood, C. Cummins and J. P. Neoptolemos (1995). "Treatment and survival in 13,560 patients with pancreatic cancer, and incidence of the disease, in the West Midlands: an epidemiological study." Br J Surg 82(1): 111-115.

8. Broberg, K., J. Björk, K. Paulsson, M. Höglund and M. Albin (2005). "Constitutional short telomeres are strong genetic susceptibility markers for bladder cancer." Carcinogenesis 26(7): 1263-1271.

9. Castelo-Branco, P., R. Leao, T. Lipman, B. Campbell, D. Lee, A. Price, C. Zhang, A. Heidari, D. Stephens, S. Boerno, H. Coelho, A. Gomes, C. Domingos, J. D. Apolonio, G. Schafer, R. G. Bristow, M. R. Schweiger, R. Hamilton, A. Zlotta, A. Figueiredo, H. Klocker, H. Sultmann and U. Tabori (2016). "A cancer specific hypermethylation signature of the TERT promoter predicts biochemical relapse in prostate cancer: a retrospective cohort study." Oncotarget 7(36): 57726-57736.

10. Cawthon, R. M. (2009). "Telomere length measurement by a novel monochrome multiplex quantitative PCR method." Nucleic Acids Res 37(3): e21. 
11. Chandrasegaram, M. D., S. C. Chiam, J. W. Chen, A. Khalid, M. L. Mittinty, E. L. Neo, C. P. Tan, P. M. Dolan, M. E. Brooke-Smith, H. Kanhere and C. S. Worthley (2015). "Distribution and pathological features of pancreatic, ampullary, biliary and duodenal cancers resected with pancreaticoduodenectomy." World Journal of Surgical Oncology 13(1): 85.

12. Chang, S., C. M. Khoo, M. L. Naylor, R. S. Maser and R. A. DePinho (2003). "Telomere-based crisis: functional differences between telomerase activation and ALT in tumor progression." Genes \& development 17(1): 88-100.

13. Ching, C. K. and J. M. Rhodes (1989). "Enzyme-linked PNA lectin binding assay compared with CA199 and CEA radioimmunoassay as a diagnostic blood test for pancreatic cancer." British journal of cancer 59(6): 949-953.

14. Distler, M., E. Pilarsky, S. Kersting and R. Grützmann (2013). "Preoperative CEA and CA 19-9 are prognostic markers for survival after curative resection for ductal adenocarcinoma of the pancreas a retrospective tumor marker prognostic study." Int J Surg 11(10): 1067-1072.

15. Dratwa, M., B. Wysoczańska, P. Łacina, T. Kubik and K. Bogunia-Kubik (2020). "TERT-Regulation and Roles in Cancer Formation." Frontiers in Immunology 11(2930).

16. El Nakeeb, A., M. El Sorogy, H. Ezzat, R. Said, M. El Dosoky, M. Abd El Gawad, A. M. Elsabagh and E. El Hanafy (2018). "Predictors of long-term survival after pancreaticoduodenectomy for periampullary adenocarcinoma: A retrospective study of 5-year survivors." Hepatobiliary Pancreat Dis Int 17(5): 443-449.

17. Feng, J., W. D. Funk, S. S. Wang, S. L. Weinrich, A. A. Avilion, C. P. Chiu, R. R. Adams, E. Chang, R. C. Allsopp, J. Yu and et al. (1995). "The RNA component of human telomerase." Science 269(5228): 1236-1241.

18. Forsmark, C. E., L. Lambiase and S. B. Vogel (1994). "Diagnosis of pancreatic cancer and prediction of unresectability using the tumor-associated antigen CA19-9." Pancreas 9(6): 731-734.

19. Haglund, C., J. Lundin, P. Kuusela and P. J. Roberts (1994). "CA 242, a new tumour marker for pancreatic cancer: a comparison with CA 19-9, CA 50 and CEA." British journal of cancer 70(3): 487492.

20. He, J., N. Ahuja, M. A. Makary, J. L. Cameron, F. E. Eckhauser, M. A. Choti, R. H. Hruban, T. M. Pawlik and C. L. Wolfgang (2014). "2564 resected periampullary adenocarcinomas at a single institution: trends over three decades." HPB (Oxford) 16(1): 83-90.

21. Hernandez-Sanchez, W., M. Xu and D. J. Taylor (2016). Chapter 21 - Telomere Maintenance and Genome Stability. Genome Stability. I. Kovalchuk and O. Kovalchuk. Boston, Academic Press: 353371.

22. Hester, C. A., E. Dogeas, M. M. Augustine, J. C. Mansour, P. M. Polanco, M. R. Porembka, S. C. Wang, H. J. Zeh and A. C. Yopp (2019). "Incidence and comparative outcomes of periampullary cancer: A population-based analysis demonstrating improved outcomes and increased use of adjuvant therapy from 2004 to 2012." J Surg Oncol 119(3): 303-317. 
23. Holt, S. E., W. E. Wright and J. W. Shay (1997). "Multiple pathways for the regulation of telomerase activity." Eur J Cancer 33(5): 761-766.

24. Hong, S.-M., C. M. Heaphy, C. Shi, S.-H. Eo, H. Cho, A. K. Meeker, J. R. Eshleman, R. H. Hruban and M. Goggins (2011). "Telomeres are shortened in acinar-to-ductal metaplasia lesions associated with pancreatic intraepithelial neoplasia but not in isolated acinar-to-ductal metaplasias." Modern Pathology 24(2): 256-266.

25. Hosen, I., P. S. Rachakonda, B. Heidenreich, P. J. de Verdier, C. Ryk, G. Steineck, K. Hemminki and R. Kumar (2015). "Mutations in TERT promoter and FGFR3 and telomere length in bladder cancer." Int J Cancer 137(7): 1621-1629.

26. Hou, L., S. A. Savage, M. J. Blaser, G. Perez-Perez, M. Hoxha, L. Dioni, V. Pegoraro, L. M. Dong, W. Zatonski, J. Lissowska, W. H. Chow and A. Baccarelli (2009). "Telomere length in peripheral leukocyte DNA and gastric cancer risk." Cancer Epidemiol Biomarkers Prev 18(11): 3103-3109.

27. Huang, D. S., Z. Wang, X. J. He, B. H. Diplas, R. Yang, P. J. Killela, Q. Meng, Z. Y. Ye, W. Wang, X. T. Jiang, L. Xu, X. L. He, Z. S. Zhao, W. J. Xu, H. J. Wang, Y. Y. Ma, Y. J. Xia, L. Li, R. X. Zhang, T. Jin, Z. K. Zhao, J. Xu, S. Yu, F. Wu, J. Liang, S. Wang, Y. Jiao, H. Yan and H. Q. Tao (2015). "Recurrent TERT promoter mutations identified in a large-scale study of multiple tumour types are associated with increased TERT expression and telomerase activation." Eur J Cancer 51(8): 969-976.

28. Jang, J. S., Y. Y. Choi, W. K. Lee, J. E. Choi, S. I. Cha, Y. J. Kim, C. H. Kim, S. Kam, T. H. Jung and J. Y. Park (2008). "Telomere length and the risk of lung cancer." Cancer Sci 99(7): 1385-1389.

29. Kamarajah, S. K. (2018). "Pancreaticoduodenectomy for periampullary tumours: a review article based on Surveillance, End Results and Epidemiology (SEER) database." Clin Transl Oncol 20(9): 1153-1160.

30. Kau, S. Y., Y. M. Shyr, C. H. Su, C. W. Wu and W. Y. Lui (1999). "Diagnostic and prognostic values of CA 19-9 and CEA in periampullary cancers." J Am Coll Surg 188(4): 415-420.

31. Kirkpatrick, K. L. and K. Mokbel (2001). "The significance of human telomerase reverse transcriptase (hTERT) in cancer." European Journal of Surgical Oncology (EJSO) 27(8): 754-760.

32. Kobitsu, K., M. Tsutsumi, T. Tsujiuchi, F. Suzuki, A. Kido, E. Okajima, T. Fukuda, T. Sakaki and Y. Konishi (1997). "Shortened telomere length and increased telomerase activity in hamster pancreatic duct adenocarcinomas and cell lines." Mol Carcinog 18(3): 153-159.

33. L., F.-C. (2001). "Periampullary carcinoma. In: Holzheimer RG, Mannick JA, editors. ." Surgical Treatment: Evidence-Based and Problem-Oriented.

34. Lee, D. D., R. Leao, M. Komosa, M. Gallo, C. H. Zhang, T. Lipman, M. Remke, A. Heidari, N. M. Nunes, J. D. Apolonio, A. J. Price, R. A. De Mello, J. S. Dias, D. Huntsman, T. Hermanns, P. J. Wild, R. Vanner, G. Zadeh, J. Karamchandani, S. Das, M. D. Taylor, C. E. Hawkins, J. D. Wasserman, A. Figueiredo, R. J. Hamilton, M. D. Minden, K. Wani, B. Diplas, H. Yan, K. Aldape, M. R. Akbari, A. Danesh, T. J. Pugh, P. B. Dirks, P. Castelo-Branco and U. Tabori (2019). "DNA hypermethylation within TERT promoter upregulates TERT expression in cancer." J Clin Invest 129(1): 223-229. 
35. Lundin, J., P. J. Roberts, P. Kuusela and C. Haglund (1994). "The prognostic value of preoperative serum levels of CA 19-9 and CEA in patients with pancreatic cancer." British journal of cancer 69(3): 515-519.

36. Maciejowski, J. and T. de Lange (2017). "Telomeres in cancer: tumour suppression and genome instability." Nature Reviews Molecular Cell Biology 18(3): 175-186.

37. McGrath, M., J. Y. Wong, D. Michaud, D. J. Hunter and I. De Vivo (2007). "Telomere length, cigarette smoking, and bladder cancer risk in men and women." Cancer Epidemiol Biomarkers Prev 16(4): 815819.

38. Mirabello, L., M. Garcia-Closas, R. Cawthon, J. Lissowska, L. A. Brinton, B. Pepłońska, M. E. Sherman and S. A. Savage (2010). "Leukocyte telomere length in a population-based case-control study of ovarian cancer: a pilot study." Cancer Causes Control 21(1): 77-82.

39. Mocellin, S., D. Verdi, K. A. Pooley, M. T. Landi, K. M. Egan, D. M. Baird, J. Prescott, I. De Vivo and D. Nitti (2012). "Telomerase reverse transcriptase locus polymorphisms and cancer risk: a field synopsis and meta-analysis." J Natl Cancer Inst 104(11): 840-854.

40. Nagtegaal, I. D., R. D. Odze, D. Klimstra, V. Paradis, M. Rugge, P. Schirmacher, K. M. Washington, F. Carneiro, I. A. Cree and W. H. O. C. o. T. E. Board (2020). "The 2019 WHO classification of tumours of the digestive system." Histopathology 76(2): 182-188.

41. Neoptolemos, J. P., R. C. Russell, S. Bramhall and B. Theis (1997). "Low mortality following resection for pancreatic and periampullary tumours in 1026 patients: UK survey of specialist pancreatic units. UK Pancreatic Cancer Group." Br J Surg 84(10): 1370-1376.

42. Oh, B. K., K. Jo Chae, C. Park, K. Kim, W. Jung Lee, K. H. Han and Y. Nyun Park (2003). "Telomere shortening and telomerase reactivation in dysplastic nodules of human hepatocarcinogenesis." $\mathrm{J}$ Hepatol 39(5): 786-792.

43. Ohtsuka, M. and M. Miyazaki (2018). "Long-term survival after resection of periampullary cancer." The Pancreas: An Integrated Textbook of Basic Science, Medicine, and Surgery: 1097-1106.

44. Okusaka, T., S. Okada, T. Sato, H. Wakasugi, H. Saisho, J. Furuse, O. Ishikawa, S. Matsuno and S. Yokoyama (1998). "Tumor markers in evaluating the response to radiotherapy in unresectable pancreatic cancer." Hepatogastroenterology 45(21): 867-872.

45. Park, S. H., J. H. Shin, K. U. Jung and S. R. Lee (2021). "Prognostic value of carcinoembryonic antigen and carbohydrate antigen 19-9 in periampullary cancer patients receiving pancreaticoduodenectomy." Asian Journal of Surgery 44(6): 829-835.

46. Posch, A., S. Hofer-Zeni, E. Klieser, F. Primavesi, E. Naderlinger, A. Brandstetter, M. Filipits, R. Urbas, S. Swiercynski, T. Jäger, P. Winkelmann, T. Kiesslich, L. Lu, D. Neureiter, S. Stättner and K. Holzmann (2020). "Hot Spot TERT Promoter Mutations Are Rare in Sporadic Pancreatic Neuroendocrine Neoplasms and Associated with Telomere Length and Epigenetic Expression Patterns." Cancers 12(6): 1625.

47. Poultsides, G. A., L. C. Huang, J. L. Cameron, R. Tuli, L. Lan, R. H. Hruban, T. M. Pawlik, J. M. Herman, B. H. Edil, N. Ahuja, M. A. Choti, C. L. Wolfgang and R. D. Schulick (2012). "Duodenal 
adenocarcinoma: clinicopathologic analysis and implications for treatment." Ann Surg Oncol 19(6): 1928-1935.

48. Rachakonda, P. S., I. Hosen, P. J. de Verdier, M. Fallah, B. Heidenreich, C. Ryk, N. P. Wiklund, G. Steineck, D. Schadendorf, K. Hemminki and R. Kumar (2013). "TERT promoter mutations in bladder cancer affect patient survival and disease recurrence through modification by a common polymorphism." Proc Natl Acad Sci U S A 110(43): 17426-17431.

49. Riall, T. S., J. L. Cameron, K. D. Lillemoe, J. M. Winter, K. A. Campbell, R. H. Hruban, D. Chang and C. J. Yeo (2006). "Resected periampullary adenocarcinoma: 5-year survivors and their 6- to 10-year followup." Surgery 140(5): 764-772.

50. Risques, R. A., T. L. Vaughan, X. Li, R. D. Odze, P. L. Blount, K. Ayub, J. L. Gallaher, B. J. Reid and P. S. Rabinovitch (2007). "Leukocyte telomere length predicts cancer risk in Barrett's esophagus." Cancer Epidemiol Biomarkers Prev 16(12): 2649-2655.

51. Ritts, R. E., Jr., D. M. Nagorney, D. J. Jacobsen, R. W. Talbot and V. R. Zurawski, Jr. (1994). "Comparison of preoperative serum CA19-9 levels with results of diagnostic imaging modalities in patients undergoing laparotomy for suspected pancreatic or gallbladder disease." Pancreas 9(6): 707-716.

52. Sandhu, V., I. M. Bowitz Lothe, K. J. Labori, O. C. Lingjærde, T. Buanes, A. M. Dalsgaard, M. L. Skrede, J. Hamfjord, T. Haaland, T. J. Eide, A. L. Børresen-Dale, T. Ikdahl and E. H. Kure (2015). "Molecular signatures of mRNAs and miRNAs as prognostic biomarkers in pancreatobiliary and intestinal types of periampullary adenocarcinomas." Mol Oncol 9(4): 758-771.

53. Sarmiento, J. M., D. M. Nagomey, M. G. Sarr and M. B. Farnell (2001). "Periampullary cancers: are there differences?" Surg Clin North Am 81(3): 543-555.

54. Satake, K. and T. Takeuchi (1994). "Comparison of CA19-9 with other tumor markers in the diagnosis of cancer of the pancreas." Pancreas 9(6): 720-724.

55. Shay, J. W. (1995). "Aging and cancer: are telomeres and telomerase the connection?" Mol Med Today 1(8): 378-384.

56. Shay, J. W. and S. Bacchetti (1997). "A survey of telomerase activity in human cancer." Eur J Cancer 33(5): 787-791.

57. Skinner, H. G., R. E. Gangnon, K. Litzelman, R. A. Johnson, S. T. Chari, G. M. Petersen and L. A. Boardman (2012). "Telomere length and pancreatic cancer: a case-control study." Cancer epidemiology, biomarkers \& prevention : a publication of the American Association for Cancer Research, cosponsored by the American Society of Preventive Oncology 21(11): 2095-2100.

58. Song, K. B., S. C. Kim, W. Lee, D. W. Hwang, J. H. Lee, J. Kwon, Y. Park, S. J. Lee and G. Park (2020). "Laparoscopic pancreaticoduodenectomy for periampullary tumors: lessons learned from 500 consecutive patients in a single center." Surg Endosc 34(3): 1343-1352.

59. Steinberg, W. (1990). "The clinical utility of the CA 19-9 tumor-associated antigen." Am J Gastroenterol 85(4): 350-355. 
60. van Heek, N. T., A. K. Meeker, S. E. Kern, C. J. Yeo, K. D. Lillemoe, J. L. Cameron, G. J. A. Offerhaus, J. L. Hicks, R. E. Wilentz, M. G. Goggins, A. M. De Marzo, R. H. Hruban and A. Maitra (2002). "Telomere shortening is nearly universal in pancreatic intraepithelial neoplasia." The American journal of pathology 161(5): 1541-1547.

61. Vilhordo, D. W., C. Gregorio, D. F. Valentini, Jr., M. I. A. Edelweiss, D. M. Uchoa and A. B. Osvaldt (2021). "Prognostic Factors of Long-term Survival Following Radical Resection for Ampullary Carcinoma." J Gastrointest Cancer 52(3): 872-881.

62. Vinagre, J., J. Nabais, J. Pinheiro, R. Batista, R. C. Oliveira, A. P. Gonçalves, A. Pestana, M. Reis, B. Mesquita, V. Pinto, J. Lyra, M. A. Cipriano, M. G. Ferreira, J. M. Lopes, M. Sobrinho-Simões and P. Soares (2016). "TERT promoter mutations in pancreatic endocrine tumours are rare and mainly found in tumours from patients with hereditary syndromes." Scientific Reports 6(1): 29714.

63. Vinothkumar, V., K. Arun, G. Arunkumar, S. Revathidevi, R. Ramani, L. Bhaskar, A. K. Murugan and A. K. Munirajan (2020). "Association between functional TERT promoter polymorphism rs2853669 and cervical cancer risk in South Indian women." Mol Clin Oncol 12(5): 485-494.

64. Willeit, P., J. Willeit, A. Mayr, S. Weger, F. Oberhollenzer, A. Brandstätter, F. Kronenberg and S. Kiechl (2010). "Telomere length and risk of incident cancer and cancer mortality." Jama 304(1): 69-75.

65. Wu, X., C. I. Amos, Y. Zhu, H. Zhao, B. H. Grossman, J. W. Shay, S. Luo, W. K. Hong and M. R. Spitz (2003). "Telomere dysfunction: a potential cancer predisposition factor." J Natl Cancer Inst 95(16): 1211-1218.

66. Xing, J., J. A. Ajani, M. Chen, J. Izzo, J. Lin, Z. Chen, J. Gu and X. Wu (2009). "Constitutive short telomere length of chromosome $17 p$ and $12 q$ but not $11 q$ and $2 p$ is associated with an increased risk for esophageal cancer." Cancer Prev Res (Phila) 2(5): 459-465.

67. Yeo, C. J., T. A. Sohn, J. L. Cameron, R. H. Hruban, K. D. Lillemoe and H. A. Pitt (1998). "Periampullary adenocarcinoma: analysis of 5-year survivors." Annals of surgery 227(6): 821-831.

68. Yuan, X., C. Larsson and D. Xu (2019). "Mechanisms underlying the activation of TERT transcription and telomerase activity in human cancer: old actors and new players." Oncogene38(34): 6172-6183.

\section{Tables:}

Due to technical limitations, tables PDF is only available as a download in the Supplemental Files section.

\section{Figures}


Figure 1

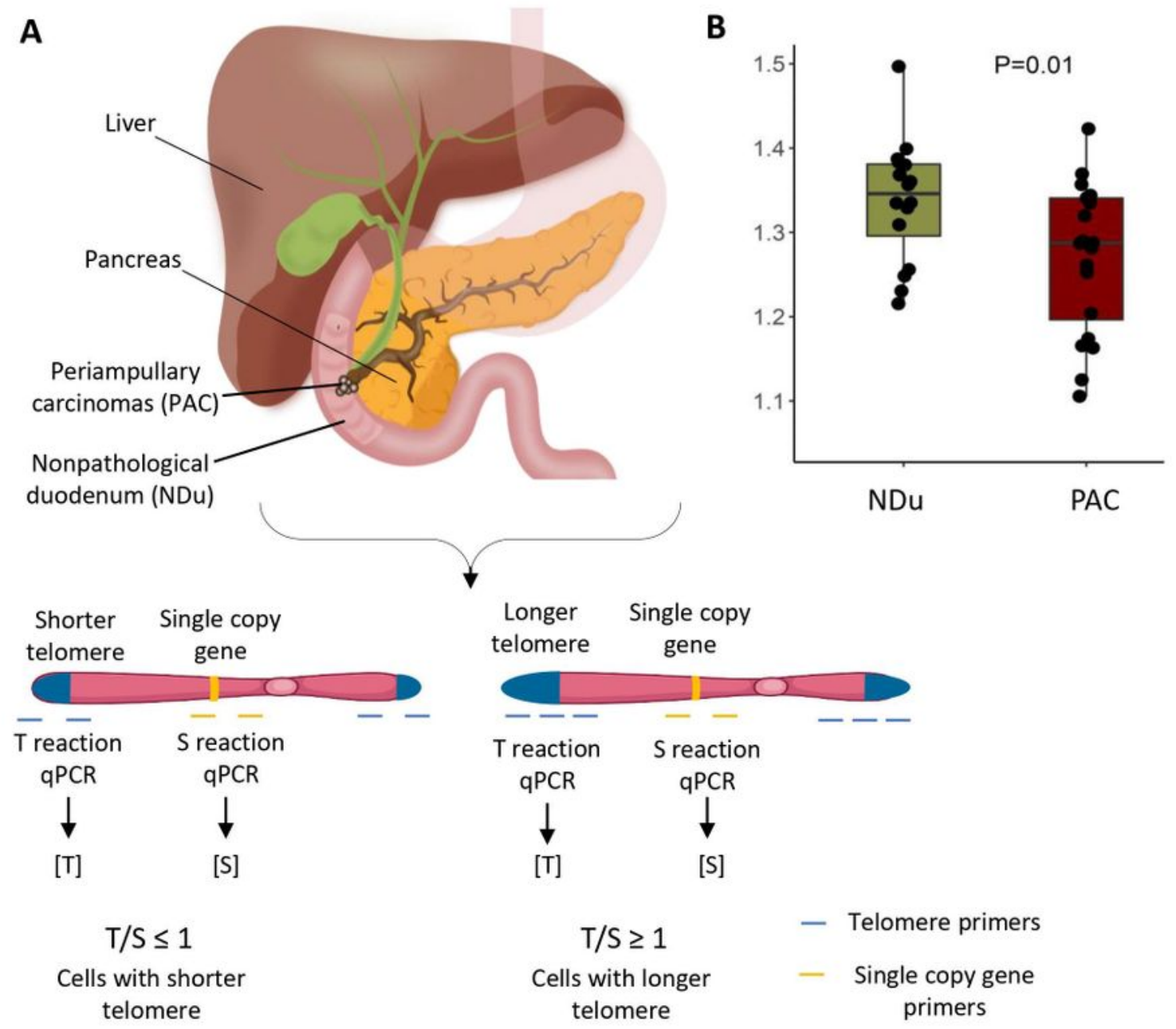

\section{Figure 1}

Relative Telomere Length (RTL) estimation. (A) Figure illustrating telomere length measurement (B) Boxplots representing the RTL of nonpathological duodenum (NDu) and periampullary carcinomas (PAC). 


\section{Figure 2}

A

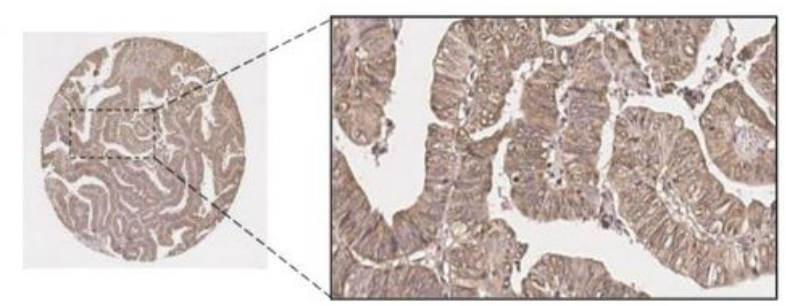

B

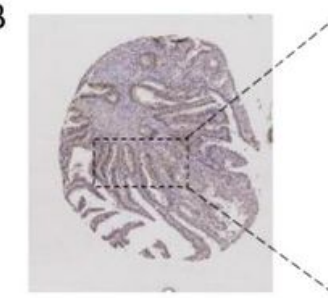

C

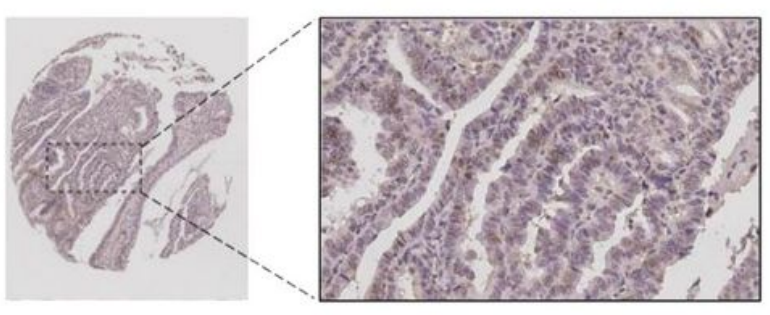

D

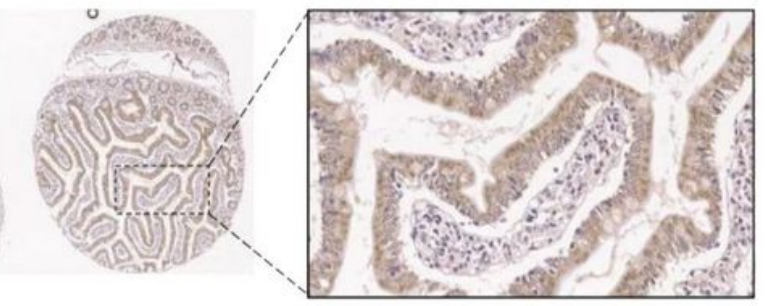

E

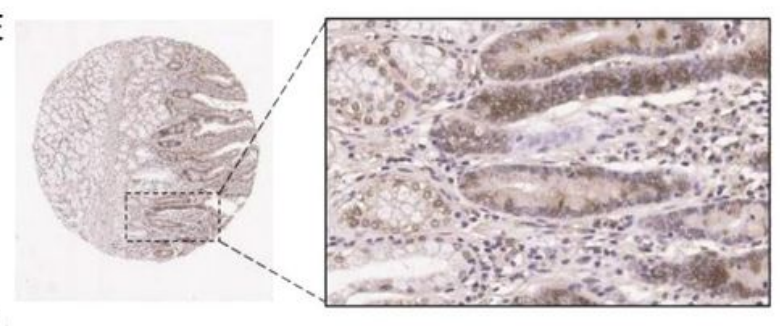

$\mathrm{F}$

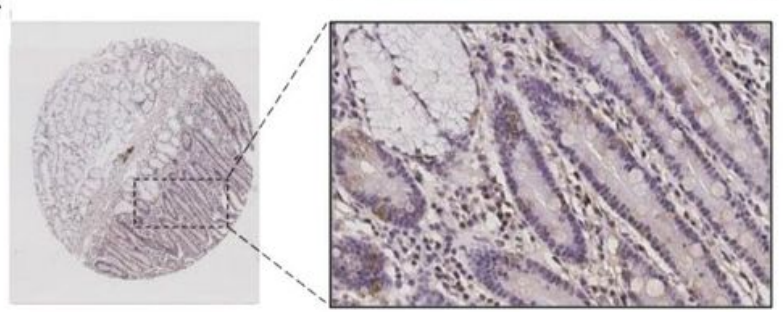

\section{Figure 2}

TERT protein expression in periampullary carcinomas (PAC) and nonpathological duodenum (NDu). (A) High expression (C3) of TERT in PAC; (B) Moderate expression (C2) of TERT in PAC, (C) Low expression (C1) of TERT in PAC, (D) High expression (C3) of TERT in NDu; (E) Moderate expression (C2) of TERT in $\mathrm{NDu}$, (F) Low expression (C1) of TERT in NDu. The histological sections stained are presented at $\times 200$ magnification and the left squares represent $x 40$ magnification. 


\section{Figure 3}

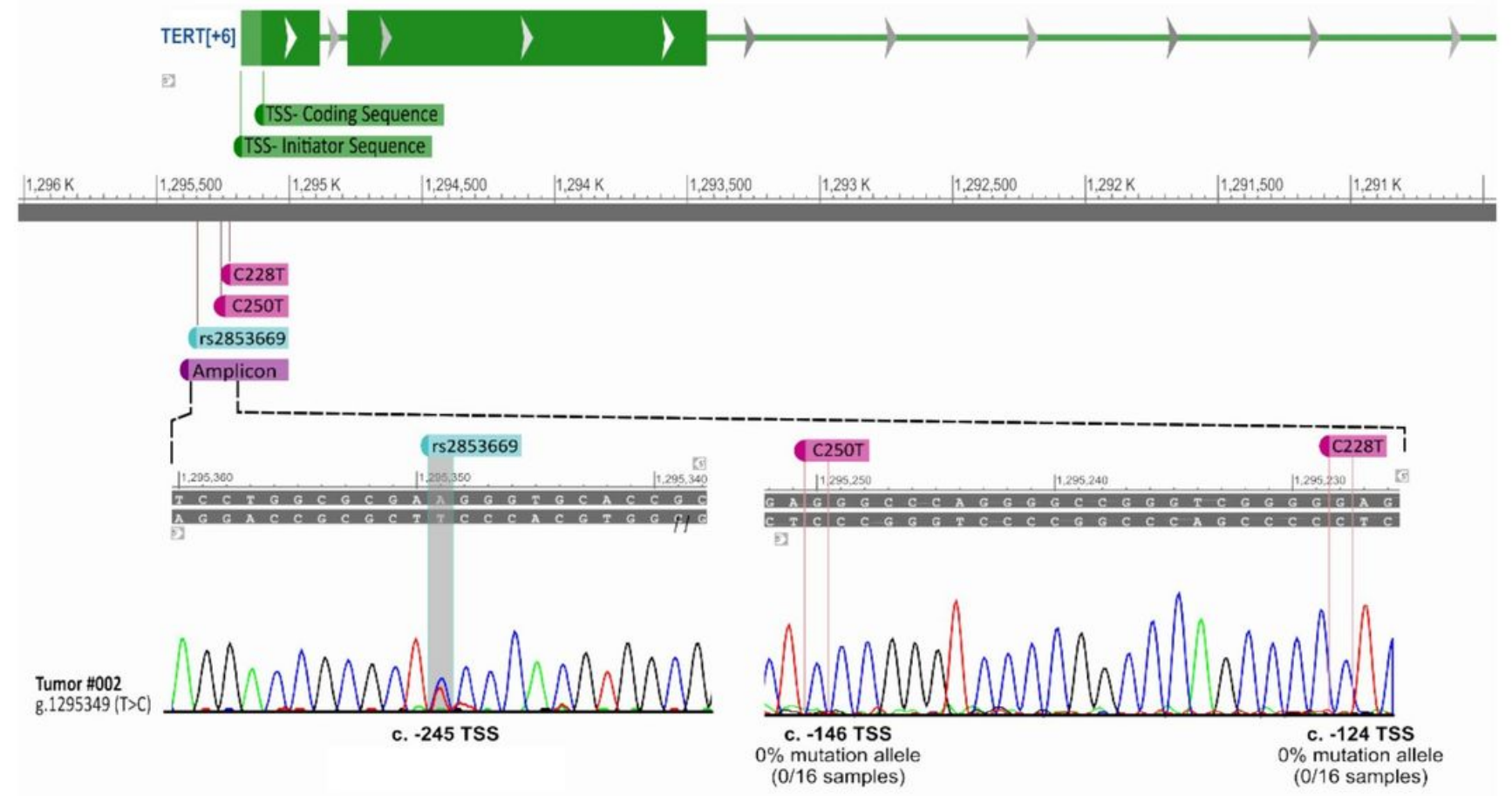

\section{Figure 3}

TERT promoter sequencing. Representative amplicon and sequencing electropherogram. Hotspot mutation site C228T (NG_009265.1:g.4935C>T) and C250T (NG_009265.1:g.4913C>T) was investigated. A polymorphic site rs2853669 (NG_009265.1: g.4814T>C) was also within the sequenced region. TSS: Transcription Start Site, reference genome: GRCh37/hg19. 


\section{Figure 4}

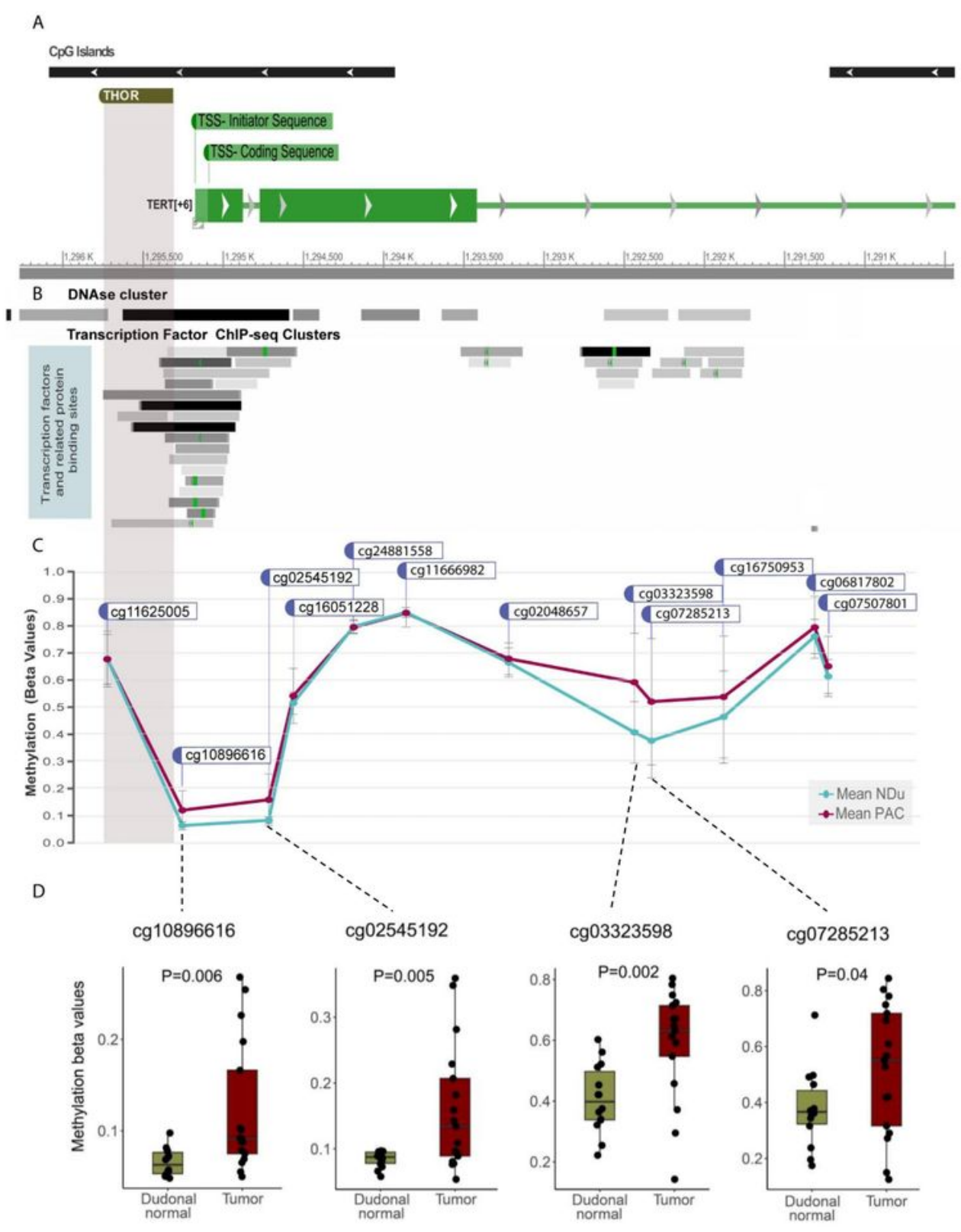

\section{Figure 4}

DNA methylation status of TERT promoter. (A) Structure of the TERT gene; (B), DNA hypersensitivity and Transcription Factors binding site (GRCh37/hg19, ENCODE); (C), Differentially methylated region (DMR) of TERT promoter (D) Box plot for CpGs within the TERT DMR. DNA Methylation is expressed as Betavalues deduced from Illumina $450 \mathrm{~K}$ arrays. 


\section{Figure 5}

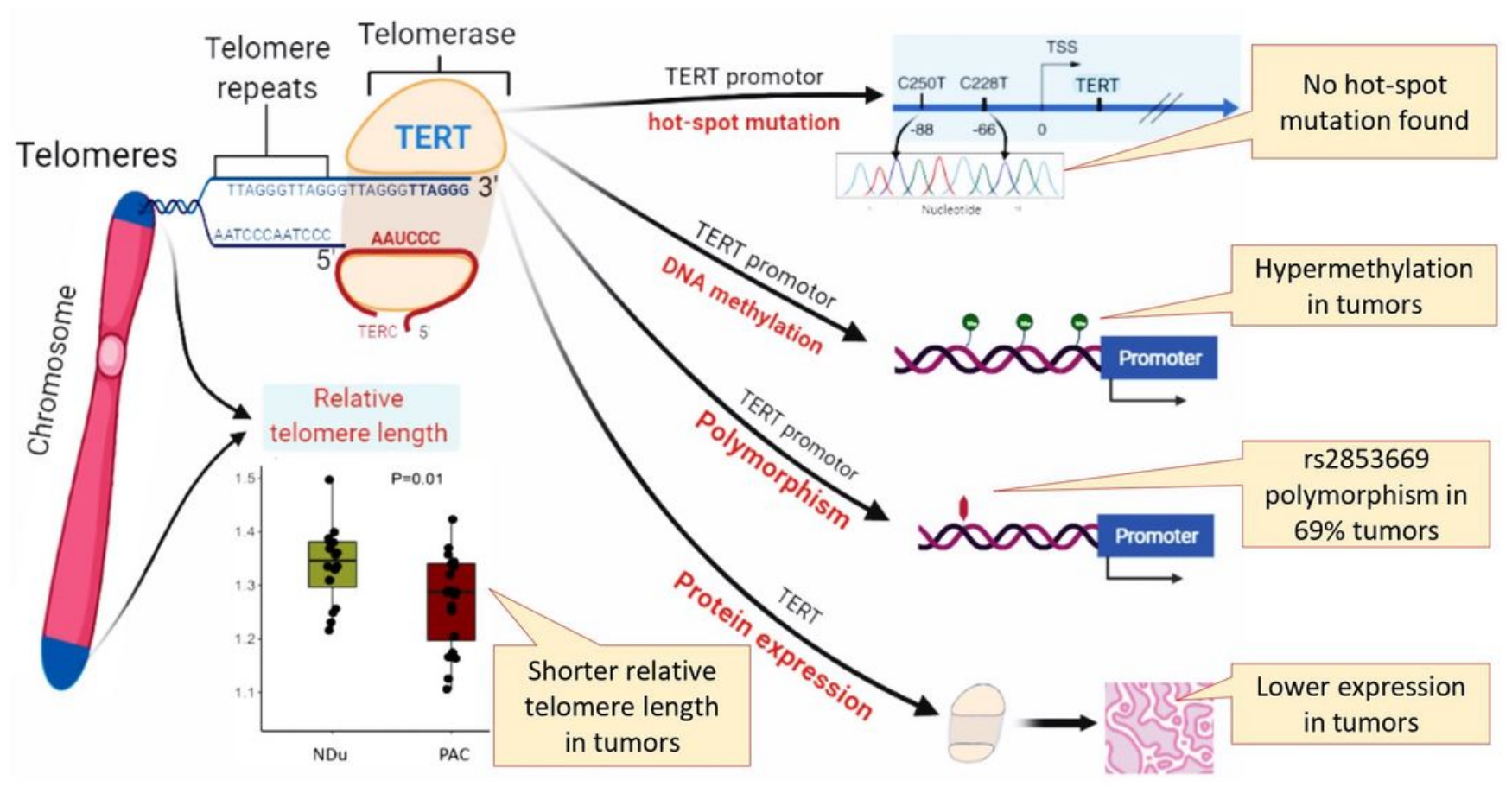

\section{Figure 5}

Result summary; showing compiled results of telomere length, TERT promoter hot-spot mutation status, TERT promoter DNA methylation status, rs2853669 SnP polymorphism in TERT promoter and TERT protein expression.

\section{Supplementary Files}

This is a list of supplementary files associated with this preprint. Click to download.

- Tables12.pdf

- Supplementarymaterialfigurestables.pdf 